\title{
Traduire
}

Revue française de la traduction

$215 \mid 2007$

La qualité en perspective

\section{TradEuras - La qualité pour une agence de traduction suisse}

\section{Philippe Golay}

\section{(2) OpenEdition}

Journals

\section{Édition électronique}

URL : http://journals.openedition.org/traduire/1327

DOI : 10.4000/traduire.1327

ISSN : 2272-9992

\section{Éditeur}

Société française des traducteurs

\section{Édition imprimée}

Date de publication : 1 décembre 2007

Pagination : 41-43

ISSN : 0395-773X

\section{Référence électronique}

Philippe Golay, «TradEuras - La qualité pour une agence de traduction suisse », Traduire [En ligne], 215 | 2007, mis en ligne le 01 décembre 2007, consulté le 30 avril 2019. URL : http:// journals.openedition.org/traduire/1327 ; DOI : 10.4000/traduire.1327 
Pour le traducteur extérieur, les mêmes qualités seront requises, à l'exception des aspects relatifs au travail en équipe et au fonctionnement de l'institution (points 6 et 7).

On attend en outre d'un collaborateur externe qu'il cherche à améliorer la qualité de ses traductions futures en tenant compte des corrections apportées à ses traductions passées par les réviseurs internes.

Sophie Marnat Chef adjoint de l'Unité française de traduction, Services linguistiques, Secrétariat général de la Banque européenne d'investissement, Luxembourg.

\section{TradEuras}

\section{La qualité pour une agence de traduction suisse}

TradEuras a élaboré une charte de qualité, dont les deux principaux axes sont :

1. La qualification et l'expérience des traducteurs, qui sont tous diplômés de cette profession et ont tous une grande pratique dans leur propre pays. Il est inutile de spécifier que ces derniers ne travaillent que vers leur langue maternelle.

2. La relecture des textes par des spécialistes des domaines des textes traduits ainsi que par des philologues professionnels de ces mêmes langues.

Ces deux axes sont articulés dans un schéma de fonctionnement selon lequel chaque texte traduit est une première fois contrôlé par un philologue de sa propre langue et une deuxième fois par un spécialiste du domaine du texte traduit, ceci avant d'être remis au traducteur initial. Le processus de chaque traduction est intégralement vérifié par le siège. 
En plus de ces deux axes principaux, la confidentialité et le respect des délais sont des éléments qui font partie des prestations de qualité que nous offrons. La charte de qualité, quant à elle, n'est pas intégrée dans les Conditions générales de vente. C'est un principe de base de TradEuras : offrir la meilleure qualité à ses clients, et ce, afin d'éviter à tout prix le schéma fatal... mais tellement logique : Peu de qualité... pas de crédibilité... donc moins de commandes !

Toute la difficulté avec des langues "rares" comme le géorgien, l'arménien ou l'azéri (azerbaidjanais) provient du fait que leur terminologie technique n'est pas aussi développée que celle des langues occidentales. Parfois trois mots sont nécessaires pour traduire un seul terme en français. Par exemple le mot "contraceptif " se traduit en azéri par hamilalik əleyhinə vasitə, littéralement " moyen contre grossesse ".

Par ailleurs, fort peu de gens en maîtrisent parfaitement les règles grammaticales et la syntaxe. Jusqu'à l'éclatement de l'URSS, la très grande majorité des textes officiels ou scientifiques n'étaient en fait élaborés qu'en russe.

À ce titre, il est impératif de trouver le correcteur qui est le plus souvent un méthodologue d'enseignement $(1)$ ou un rédacteur professionnel dans un journal de l'une de ces langues et qui ne laissera pas passer une faute.

En ce qui concerne les traductions techniques, la double relecture par un professionnel du domaine du texte est systématique. Il est arrivé, dans quelques cas, que nous connaissions un traducteur externe qui soit au départ un professionnel du domaine spécifique du texte à traduire et que, par conséquent, nous n'ayons pas eu à effectuer une double relecture en plus de la correction linguistique. Lorsqu'il s'agit de textes simples, pour lesquels le client a préalablement et clairement exprimé n'avoir aucune exigence de qualité, nous ne soumettons pas non plus la traduction à un double filtrage.

(1) Didacticien et enseignant, NdlR. 
L'important est de toujours procéder avec le client à une analyse préalable de ses besoins et d'adapter nos prestations en conséquence. Il arrive qu'une traduction de qualité parfaite ne corresponde pas à ses attentes et à ses exigences, ou qu'il ne souhaite avoir qu'une "idée générale " de ce qui figure dans le texte. Ceci est le plus souvent valable pour les traductions des langues rares vers le français.

Par révision, nous entendons un travail spécifique de contrôle qui est effectué lorsqu'un texte, précédemment traduit par un tiers, fait l'objet d'une demande de vérification par un client. Comme pour la traduction, interviennent le traducteur, le linguiste ainsi qu'une personne spécialisée dans le domaine du texte traduit.

Si la traduction vers des langues rares implique moins de fournisseurs, elle se conjugue également avec moins de demandes de traduction que pour les langues européennes, pour lesquelles il y a un nombre considérables de traducteurs externes spécialisés dans tel ou tel domaine. Pour de telles commandes, TradEuras n'est pas compétente mais fait appel en France ou en Suisse à des professionnels de jure, dont certains font partie de la SFT et envers lesquels nous avons toute confiance !

$\mathrm{Si}$, aujourd'hui, les demandes en traduction du français vers les langues rares de l'Union européenne sont encore peu fréquentes, nous avons tout lieu de penser que l'élargissement progressif de l'Europe vers l'Est suscitera de nouveaux besoins ces prochaines années, que ce soit dans les domaines juridique, commercial, scientifique ou culturel.

Seul l'avenir nous le dira...

Philippe Golay

Agence de traduction TradEuras - Français/Langues Euro-asiatiques tradeuras@vtxnet.ch 